\title{
Adjustment of Sampling Locations for Inspected Datasets to Observe Variations in Track Geometry over Time
}

\author{
Masako KAMIYAMA, Ph.D. \\ Senior Researcher, \\ Track Geometry \& Maintenance Laboratory, Track Technology Division
}

\begin{abstract}
This article describes a procedure for adjusting sampling locations in inspected datasets of railway track geometry. These sets are spatially discretized and their sampling locations change with each measurement, meaning that variations in track geometry cannot be simply computed as the difference between two datasets. The difficulty of the adjustment arises from the fact that some sampling intervals locally shorten and lengthen. In the proposed procedure, the adjustment is formulated into an optimization form, and the one unknown parameter involved in the form is stochastically identified. Consequently, the variation datasets are obtained and they demonstrate unknown properties.
\end{abstract}

Keywords: track inspection, dynamic programming, maximum likelihood method

\section{Introduction}

To maintain the geometry of railway tracks, special rolling stock known as a track inspection car periodically measures the track geometry, which varies slightly under the loads of passing trains. Such geometric variations must be maintained within prescribed limits to keep trains running safely. While running on the rails, the inspection car continuously measures a set of geometric aspects such as the vertical and horizontal geometry and the distance between the two rails (known as the gauge). These geometric measurements are simultaneously discretized at fixed spatial intervals and are recorded as digital datasets as illustrated in Fig. 1.

These measurements are used in planning track maintenance schedules as well as in evaluating existing rail conditions. From the viewpoint of planning, future geometry must be predicted from previous measurements obtained on the same track. Although it is desirable that these discretized locations can be fixed to observe changes in track geometry, the actual position of sam-

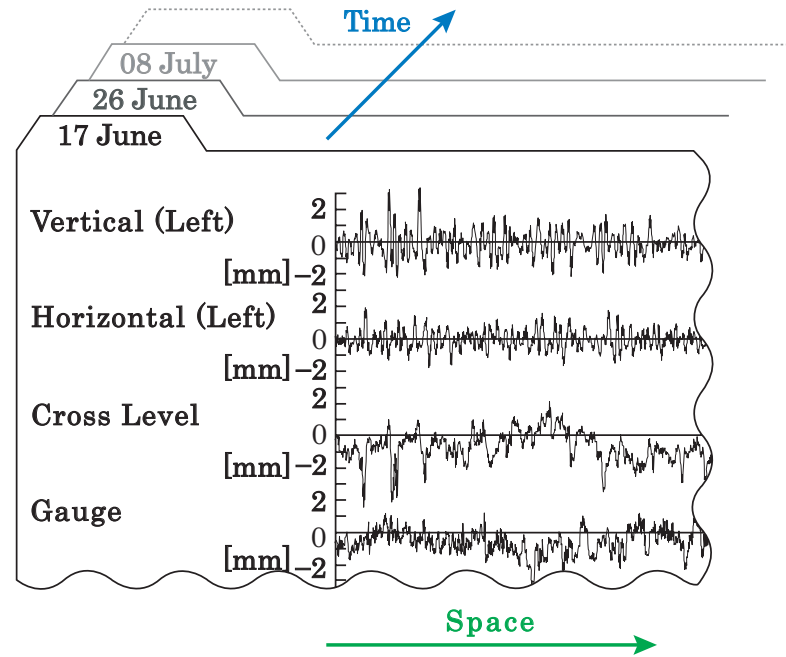

Fig. 1 Scheme for measurement of railway track geometry and its database pling locations on rails changes slightly with each measurement, as illustrated in Fig. 2. These changes are caused by the pulse used for selecting sampling locations (i.e. the wheel-rotation pulse) being linked to the rotation of the car wheel, as illustrated in Fig. 3. Identical spatial discretization cannot therefore be reproduced.

Moreover, it is difficult to adjust these location gaps after the discretization. If the spatial intervals (i.e. the sampling interval) are kept constant for two measuring runs, these gaps could be easily adjusted by calculating the correlation coefficient distance between the two datasets, even if the locations themselves change. However, in reality, some sampling intervals may locally shorten or lengthen due to irregular rotation of the car wheel or malfunction of the sampling device. Unfortunately, the length and location of these locally irregular intervals cannot be detected, which causes difficulty in

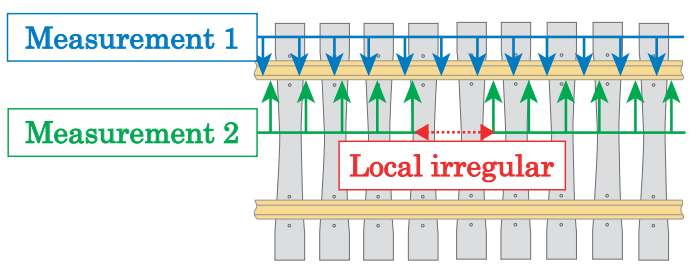

Fig. 2 Scheme for discretized-location gaps on railway track for two measurements

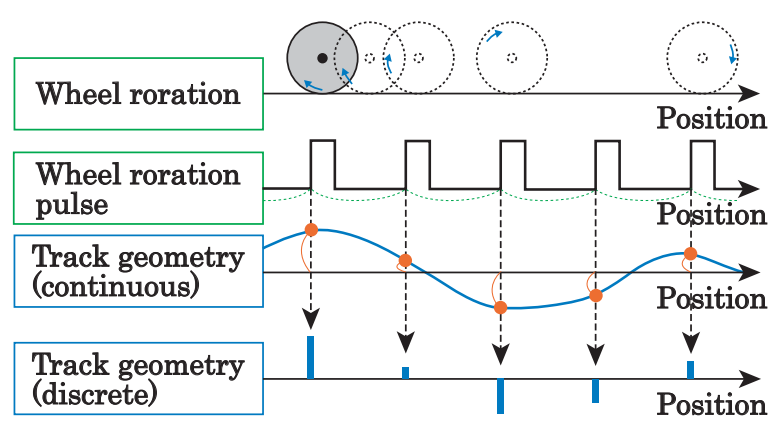

Fig. 3 Scheme for spatially discretized geometry of railway tracks with wheel-rotation pulses 
adjusting the location gaps.

An example of irregular sampling intervals is shown in Fig. 4. The two upper curves (A) and (B) show the values of track gauges measured in the same section of track, and both demonstrate similar behaviour, since the track gauge hardly varies under the load of passing trains. The difference sequences between (A) and (B) are also shown below. Note that a difference operation is performed on different intervals of (A). The amplitude of both difference sets changes abnormally in the second half, although the two gauge curves appear similar. Some sampling intervals therefore appear to shorten or lengthen locally during one of the two measuring runs.

Conventionally, variations in track geometry have been evaluated and predicted only by comparing representative statistics such as maximum values. This is because such variations could not be computed from the differences between the two datasets obtained using a track inspection car owing to the location problem outlined above. When these statistics are computed, the measured datasets are cut to sections of a given length to allow representative statistics to be obtained. Prediction of future geometry from the representative statistics computed by this method assumes that the mechanical properties influencing geometric changes are piecewise constant in the given section. As an example, it is assumed that each datum in the same section is selected at random from a normal distribution.

The critical point is that predictions based on this assumption are too rough to enable prediction of geometric changes at vulnerable points on the railway track and to allow analysis of the mechanisms that influence such changes. First, railway tracks generally undergo structural alterations (such as at level crossings and bridges) where mechanical uniformity is broken. Moreover, these boundaries also tend to represent points of vulnerability and require accurate prediction. Second, the piecewise analysis outlined above cannot reveal the mechanical interaction that changes track geometry among different points on the same track. Being able to compute geometric changes for every sampling location would be helpful in understanding the various mecha-

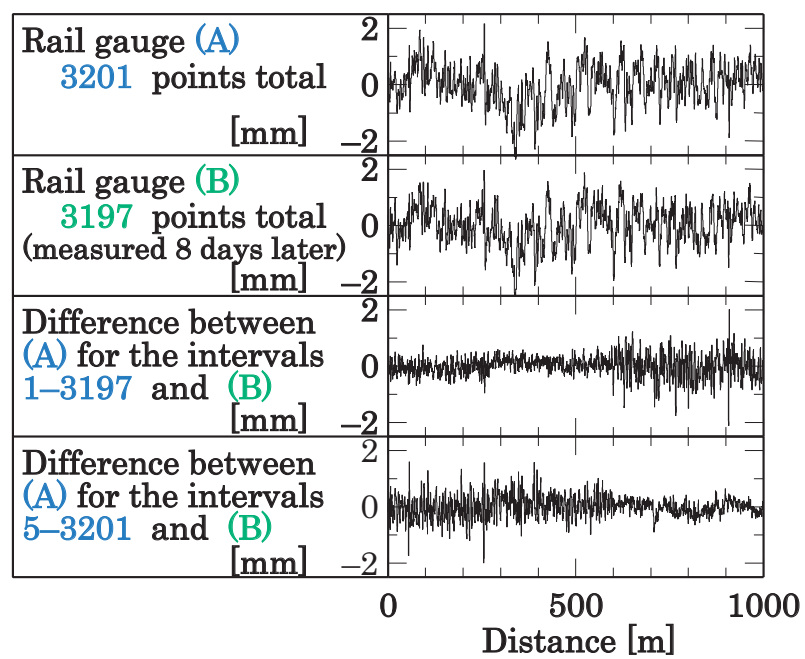

Fig. 4 Example of datasets from two measurements for the same section of track and their differences

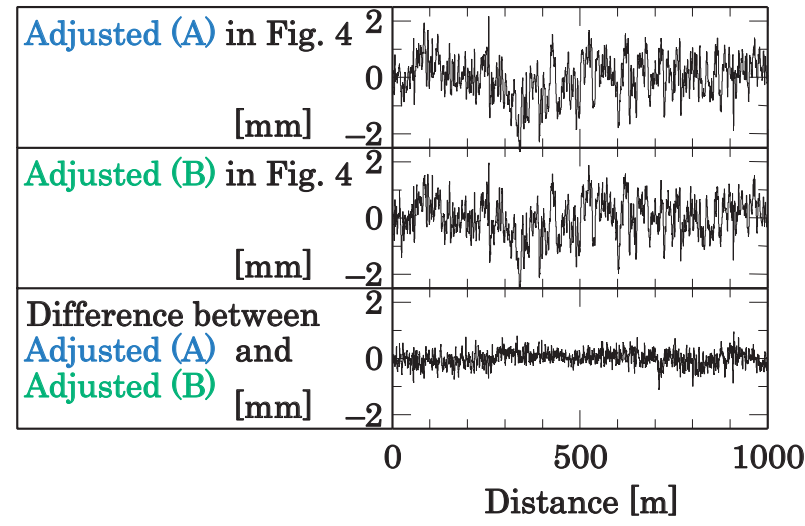

Fig. 5 Example of proposed adjustment. The original datasets of $(A)$ and $(B)$ are shown in Fig.4.

nisms that influence track geometry.

The author has therefore developed a procedure for adjusting the sampling locations in two or more datasets measured using a track inspection car. Figure 5 shows an example of adjusting the locations with the proposed procedure. The abnormal amplitude (seen in the difference datasets in Fig. 4) is suppressed, although the two gauge curves still demonstrate similar behaviour. The proposed procedure reveals that the wheel slid approximately one meter when the dataset (B) was inspected.

The next section introduces modelling for the causes of gaps and the method for adjustment. Section 3 presents a solution to the problem, and Section 4 demonstrates some applications of this method. Finally, Section 5 provides conclusions.

\section{Transformation from proposed adjustment into a searching problem}

\subsection{Basic principle of proposed adjustment}

The author decided to adjust the sampling locations with reference to one item of track geometry, although the rail inspection car simultaneously measures several geometric items. Sampling locations of the other datasets, which had been simultaneously inspected with the adjusted dataset, were then shifted according to the primary adjustment. This was done because the adjustment needs to utilize assumptions regarding temporal variations in track geometry. This is not ideal, since the author aims to predict the future geometry itself after the proposed adjustment. Therefore the procedure should also involve minimal assumptions.

To satisfy this condition, the dataset of the gauge (Fig. 6) is the most useful since it seems to show the least variation under train loads. This selection assumes that temporal variations in the gauge are negligible in the adjustment.

To simplify the adjustment procedure, it was decided that the gauge datasets to be adjusted should satisfy the following two conditions:

- Each dataset should be extracted from the long set originally measured. In addition, both ends of the extracted set should respectively correspond to two cer- 


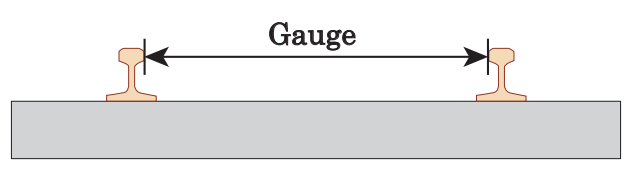

Fig. 6 Illustration of rail gauge

tain positions fixed on the railway track.

- Maintenance operations should not be performed on the track between the measurements of any two datasets.

The first condition ensures that the two extracted datasets roughly match each other. This condition can be satisfied because some data points in the originally measured dataset roughly match the points fixed on the railway track. These points are identified by peaks in the pulse signals generated by the transmitters embedded in the railway track and detected using the inspection car, as illustrated in Fig. 7. This pulse, known as a positioning pulse, is simultaneously recorded and discretized with the other measured signals. After discretization, the peaks of the discrete positioning pulse indicate that the corresponding data points are equivalent to the positions above the ground transmitters.

However, these peaks do not indicate the positions exactly above the transmitters, since the peak of the signal is held before the spatial discretization, as illustrated in Fig. 7. This holding is performed to prevent the loss of these peaks with the discretization. The peak therefore corresponds to the first location where the wheelrotation pulse was generated after the detector in the car passed the transmitter.

The second condition ensures that changes in the track geometry are negligible, since track maintenance work noticeably changes track geometry (including the gauge) whereas train loads produce only slight changes. Thus, only noise generated from the measuring device should affect the adjustment under this condition.

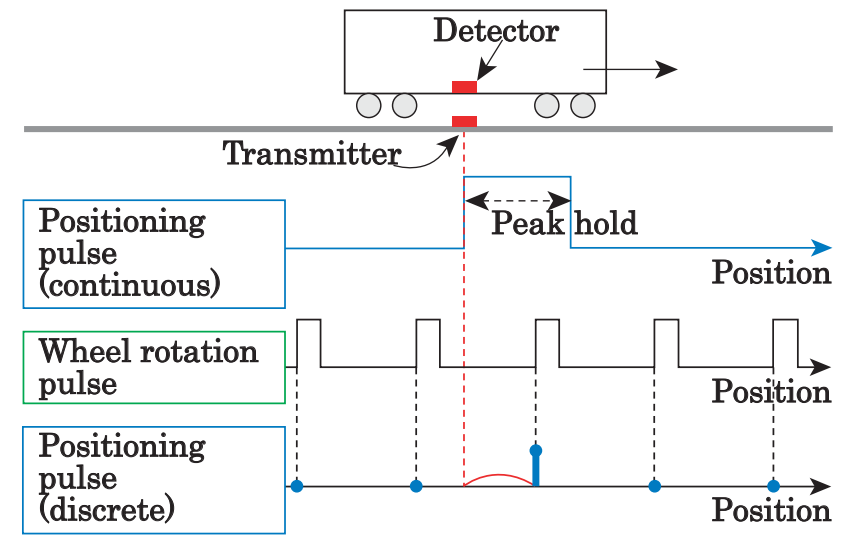

Fig. 7 Scheme for positioning-pulse signal generated on railway track and discretized with wheel-rotation pulse

\subsection{Transformation of proposed adjustment}

The sampling locations for two datasets were adjusted according to the following procedure, as illustrated in Fig. 8:

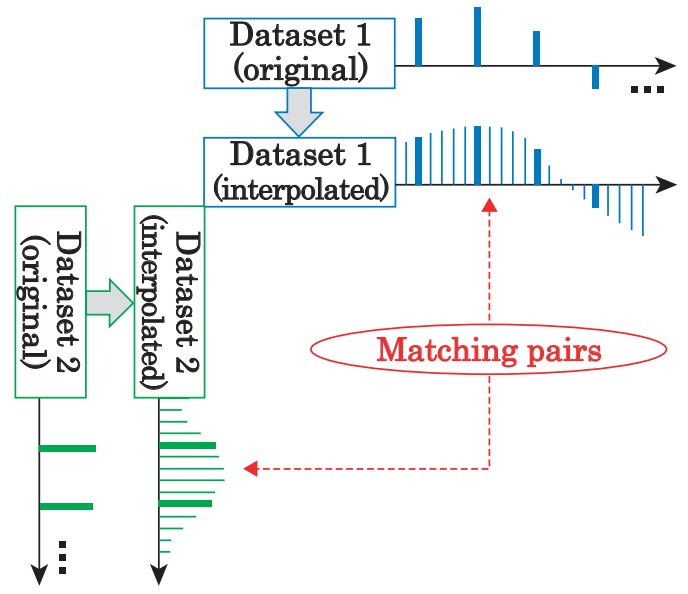

\section{Fig. 8 Scheme of basic idea for adjusting discretized- location gaps}

1. The original sampling intervals of both datasets are divided by positive integer $\alpha$, and the values for the newly generated dataset are obtained through interpolation.

2. Data points corresponding to each other are selected. Thus, this procedure yields datasets whose sampling intervals are shortened to a fraction of $\alpha$ after adjustment.

Step 1 in the above procedure approximates the values of the data newly generated by interpolation so as not to change the frequency characteristic of the original dataset [1], since a low-pass filter has already smoothed the datasets before discretization. In other words, this filter assures that the dataset just before the spatial discretization does not contain higher-frequency components.

In this procedure, the adjustment eventually results in the construction of sequences composed of interpolated datasets. When two datasets are to be adjusted, this procedure establishes correspondence between the $m_{t}$ th data in one dataset and the $n_{t}$ th data in another (as illustrated in Fig. 9) where $t$ is the number of the pair. Thus, the adjustment results in the construction of a sequence composed of the data-point indices $\left\{m_{1}, m_{2}, \ldots, m_{T}\right\}$ and $\left\{n_{1}, n_{2}, \ldots, n_{T}\right\}$, where $T$ is the total number of pairs, such that $1 \leq t \leq \mathrm{T}$.

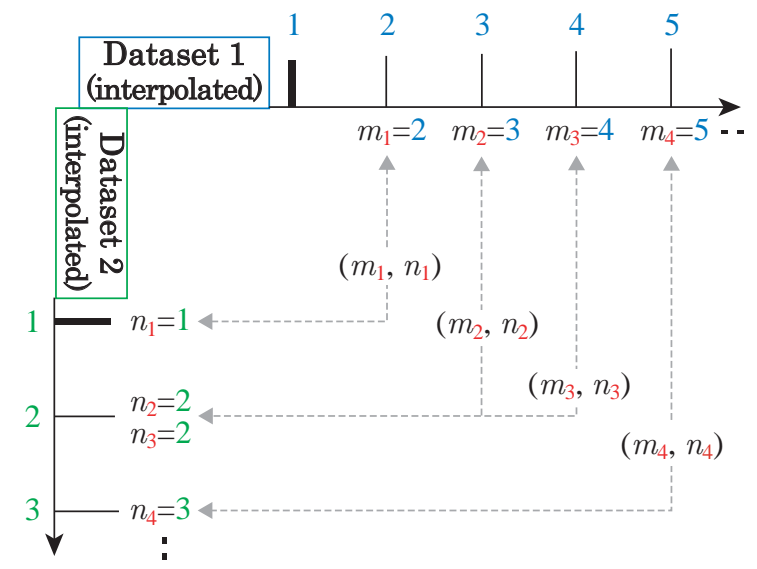

Fig. 9 Notation of sequences $\left\{m_{1}, m_{2}, \ldots, m_{T}\right\}$ and $\left\{n_{1}, n_{2}, \ldots, n_{T}\right\}$ composed of data-point indices 
The sequences $\left\{m_{1}, m_{2}, \ldots, m_{T}\right\}$ and $\left\{n_{1}, n_{2}, \ldots, n_{T}\right\}$ must satisfy the following conditions:

- $1 \leq m_{1} \leq m_{2} \leq \cdots \leq m_{T} \leq M$, where $M$ represents the number of the data contained in one dataset after interpolation.

- $1 \leq n_{1} \leq n_{2} \leq \cdots \leq n_{T} \leq N$, where $N$ represents the number of the data contained in the other dataset after interpolation.

- $\left\{m_{1}, m_{2}, \ldots, m_{T}\right\}$ and $\left\{n_{1}, n_{2}, \ldots, n_{T}\right\}$ are all positive integers.

- $0 \leq m_{i}-m_{i-1} \leq 1$ for all $i \geq 2$.

- $0 \leq n_{i}-n_{i-1} \leq 1$ for all $i \geq 2$.

- $m_{i}-m_{i-1}=1$ and/or $n_{i}-n_{i-1}=1$ for all $i \geq 2$.

- $m_{1}=1$ and/or $n_{1}=1$.

- $m_{T}=M$ and/or $n_{T}=N$.

Note that several sequences obviously satisfy the above conditions, meaning that a number of feasible solutions exist. The optimal sequence must therefore be selected from the feasible ones.

\section{Selection of optimal data-point sequence}

This section describes the procedure for selecting the sequence constructed using all the optimal data points $\left\{\tilde{m}_{1}, \tilde{m}_{2}, \ldots, \tilde{m}_{T}\right\}$ and $\left\{\tilde{n}_{1}, \tilde{n}_{2}, \ldots, \tilde{n}_{T}\right\}$ from all the feasible sets $\left\{m_{1}, m_{2}, \ldots, m_{T}\right\}$ and $\left\{n_{1}, n_{2}, \ldots, n_{T}\right\}$. First, a form that can evaluate the feasible sets is formulated as in Section 3.1. This evaluation function can be successfully optimized through dynamic programming. However, the function involves the unknown parameter $e$ that affects the optimization. In Section 3.2, the parameter $e$ is therefore stochastically identified using the maximum likelihood method.

Note that $\left\{m_{1}, m_{2}, \ldots, m_{T}\right\}$ and $\left\{\tilde{m}_{1}, \tilde{m}_{2}, \ldots, \tilde{m}_{T}\right\}$ are denoted below by $\mathbf{m}_{1: T}$ and $\tilde{\mathbf{m}}_{1: T}$ respectively. The other variables are similarly expressed to demonstrate the differences between a sequence and a term.

\subsection{Formulation of adjustment as an optimization problem}

The solution that minimizes the following constrained nonlinear function, which can evaluate $\left(\mathbf{m}_{1: T}, \mathbf{n}_{1: T}\right)$, is denoted by $\left(\tilde{\mathbf{m}}_{1: T}, \tilde{\mathbf{n}}_{1: T}\right)$ :

$$
\begin{aligned}
& \left(\tilde{\mathbf{m}}_{1: T}, \tilde{\mathbf{n}}_{1: T}\right) \\
= & \arg \min F\left(\mathbf{m}_{1: T}, \mathbf{n}_{1: T}\right) \\
= & \underset{\mathbf{m}_{1: T}, \mathbf{n}_{1: T}}{\arg \min }\left\{\sum_{i=1}^{T}\left[f\left(m_{i}\right)-g\left(n_{i}\right)\right]^{2}+e \sum_{i=2}^{T}\left[\delta\left(m_{i}-m_{i-1}\right)-\delta\left(n_{i}-n_{i-1}\right)\right]\right\},
\end{aligned}
$$

where

- $F\left(\mathbf{m}_{1: T}, \mathbf{n}_{1: T}\right)$ is a function that evaluates $\mathbf{m}_{1: T}$ and $\mathbf{n}_{1: T}$.

- $f\left(m_{i}\right)$ is the value of the $m_{i}$ th data in one dataset.

- $g\left(n_{i}\right)$ is the value of the $n_{i}$ th data in the other dataset.

- $e \geq 0$ is a weighting coefficient.

- $\delta(\cdot)$ is a function defined as

$$
\delta(x)= \begin{cases}1, & x=0, \\ 0, & x \neq 0 .\end{cases}
$$

The details of the two terms $\sum_{i=1}^{T}\left[f\left(m_{i}\right)-g\left(n_{i}\right)\right]^{2}$ and $e \sum_{i=2}^{T}\left[\delta\left(m_{i}-m_{i-1}\right)-\delta\left(n_{i}-n_{i-1}\right)\right]$ are given below.
The first term $\sum_{i=1}^{T}\left[f\left(m_{i}\right)-g\left(n_{i}\right)\right]^{2}$ evaluates the residual sequence computed when the two sequences $\mathbf{m}_{1: T}$ and $\mathbf{n}_{1: T}$ are all given. Although a smaller summation naturally indicates a better fit, a data-point index sequence cannot be reasonably obtained using only this summation term. Figure 10 shows the results of minimization if the evaluation function $F\left(\mathbf{m}_{1: T}, \mathbf{n}_{1: T}\right)$ consists of only this first term (equivalent to $e=0$ in (1)). Obviously this result behaves unreasonably, since $T$ is much larger than $M$ and $N$, and the two adjusted sequences excessively fit for each other. This is because many data are unnecessarily reused more than twice to adjust the sampling intervals.

The second term $e \sum_{i=2}^{T}\left[\delta\left(m_{i}-m_{i-1}\right)-\delta\left(n_{i}-n_{i-1}\right)\right]$ eliminates the excessive fit mentioned above, since the weighting coefficient $e \geq 0$ is added to the evaluation function each time the same datum is reused. Naturally, when the parameter $e$ has a larger value, each sampling interval is more difficult to shorten or lengthen.

Although this evaluation function can be optimized using the dynamic programming technique, the function itself depends on the unknown parameter $e$. It is therefore necessary to carefully identify $e$ before the optimization.

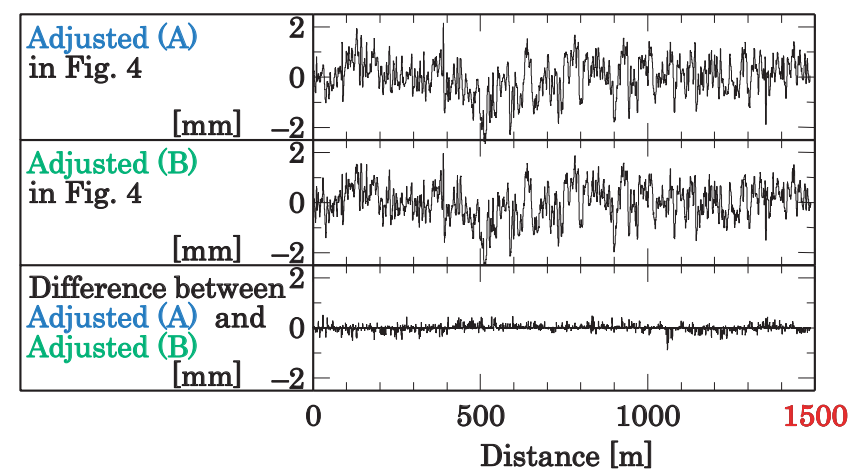

Fig. 10 Example of adjustment when $e=0$. The original datasets of $(A)$ and $(B)$ are shown in Fig.4

\subsection{Interpretation of optimized sequence}

In order to identify a suitable value for $e$, evaluation function (1) is stochastically interpreted. In (1), it is assumed that $f\left(\tilde{\mathbf{m}}_{1: T}\right)-g\left(\tilde{\mathbf{n}}_{1: T}\right)$ is a normal random variable with zero mean and variance $\sigma^{2}$. By multiplying (1) with $-1 /\left(2 \sigma^{2}\right)$ and exponentiating it, (1) can be transformed into

$$
\begin{aligned}
& \left(\tilde{\mathbf{m}}_{1: T}, \tilde{\mathbf{n}}_{1: T}\right) \\
= & \underset{\mathbf{m}_{1: T}, \mathbf{n}_{1: T}}{\arg \min }\left\{\prod_{i=1}^{T} \exp \left[-\frac{1}{2 \sigma^{2}}\left(f\left(m_{i}\right)-g\left(n_{i}\right)\right)^{2}\right]\right\} \\
& \times \exp \left\{-\frac{e}{2 \sigma^{2}} \sum_{i=2}^{T}\left[\delta\left(m_{i}-m_{i-1}\right)+\delta\left(n_{i}-n_{i-1}\right)\right]\right\} .
\end{aligned}
$$

The right side of (2) is divided into two parts with \{\} , and each part has its own interpretation. The first part is proportional to the likelihood measure of $f\left(\mathbf{m}_{1: T}\right)-g\left(\mathbf{n}_{1: T}\right)$, when $\mathbf{m}_{1: T}$ and $\mathbf{n}_{1: T}$ are given. On the other hand, the second part is proportional to the probability distribution of $\left(\mathbf{m}_{1: T}, \mathbf{n}_{1: T}\right)$. This distribution is defined without referring to any value in the original datasets $(f(1: M), g(1: N))$. 
Hence, the process of obtaining $\left(\tilde{\mathbf{m}}_{1 \cdot T}, \tilde{\mathbf{n}}_{1 \cdot T}\right)$ from (1) is equivalent to searching a sequence that maximizes the probability of $\left(\mathbf{m}_{1: T}, \mathbf{n}_{1: T}\right)$ in its probability distribution with reference to all values $(f(1: M), g(1: N))$, i.e. the new interpretation of evaluation function (1). Note that the probability distribution of $\left(\mathbf{m}_{1: T}, \mathbf{n}_{1: T}\right)$ is specified by the parameter $e$.

Thus, $e$ can be evaluated using the likelihood function $L(e)$ [2], which is defined as a likelihood of the distribution of $f\left(\mathbf{m}_{1: T}\right)-g\left(\mathbf{n}_{1: T}\right)$. The most suitable parameter $e$ most closely approaches this distribution to the normal distribution $N\left(0, \sigma^{2}\right)$. In other words, the best value of $e$ represents the best stochastic model shown in (2). This technique, which evaluates parameters using a likelihood value, is known as the maximum likelihood method. Due to space constraints, this article does not present the definition of $L(e)$ or the method for computing a suitable value of $e$ [3].

The optimal result of adjustment using $e$ identified by the maximum likelihood method has been previously shown in Fig. 5. The non-stationary behaviour seen in the residual sequence before adjustment disappears. The value of $e$ also changes the distribution of the residuals obtained with the adjustment, as shown in Fig. 11. The residuals in Fig. 5 seem to obey standard normal distribution because of the identified $e$ value, although the residuals in Fig. 10 are highly concentrated around zero because $e=0$.

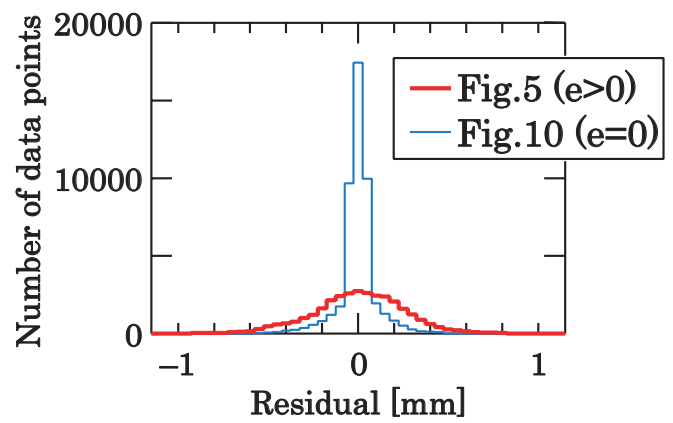

Fig. 11 Value distribution of residuals shown in Figs. 5 and 10

\section{Application and discussion}

In this section, several gauge datasets selected from those shown in Fig. 12 are adjusted using the developed procedure. These 11 gauge datasets were measured in the same section of track over a period of about four months, and all the curves seem to exhibit similar behaviour.

The procedural steps for adjustment based on the method outlined are described below:

1. The measured datasets sandwiched between two positioning pulses, which indicate the positions of transmitters fixed on the railway tracks, are extracted.

2. All the datasets are browsed, and those for adjustment are selected.

3. The selected datasets are interpolated.

4. The optimal value of $e$ is evaluated using the maximum likelihood method.

5. The sequences composed of the data-point indices are

\begin{tabular}{|c|c|c|}
\hline $\begin{array}{l}\text { Date: } 17 \text { June } \\
3197 \text { points total } \\
\text { Car } 1 \quad[\mathrm{~mm}]\end{array}$ & $\begin{array}{r}2 \\
0 \\
-2 \\
\end{array}$ & 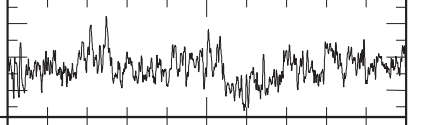 \\
\hline $\begin{array}{l}\text { Date: } 26 \text { June } \\
3198 \text { points total } \\
\text { Car } 1 \quad \text { [mm] }\end{array}$ & $\begin{array}{r}2 \\
0 \\
-2 \\
\end{array}$ & W \\
\hline $\begin{array}{l}\text { Date: } 08 \text { July } \\
3197 \text { points total } \\
\text { Car } 2 \quad[\mathrm{~mm}]\end{array}$ & $\begin{array}{r}2 \\
0 \\
-2 \\
\end{array}$ & \\
\hline $\begin{array}{l}\text { Date: } 18 \text { July } \\
3198 \text { points total } \\
\text { Car } 2 \quad[\mathrm{~mm}]\end{array}$ & $\begin{array}{r}2 \\
0 \\
-2 \\
\end{array}$ & \\
\hline $\begin{array}{l}\text { Date: } 27 \text { July } \\
3198 \text { points total } \\
\text { Car } 2 \quad[\mathrm{~mm}]\end{array}$ & $\begin{array}{r}2 \\
0 \\
-2 \\
\end{array}$ & \\
\hline $\begin{array}{l}\text { Date: } 05 \text { August } \\
3198 \text { points total } \\
\text { Car } 1 \quad \text { [mm] }\end{array}$ & $\begin{array}{r}2 \\
0 \\
-2 \\
\end{array}$ & \\
\hline $\begin{array}{l}\text { Date: } 17 \text { August } \\
\text { 3198 points total } \\
\text { Car } 1 \quad \text { [mm] }\end{array}$ & $\begin{array}{r}2 \\
0 \\
-2 \\
\end{array}$ & \\
\hline $\begin{array}{l}\text { Date: } 26 \text { August } \\
3198 \text { points total } \\
\text { Car } 2 \quad[\mathrm{~mm}]\end{array}$ & $\begin{array}{r}2 \\
0 \\
-2 \\
\end{array}$ & Exy \\
\hline $\begin{array}{l}\text { Date: } 04 \text { September } \\
3198 \text { points total } \\
\text { Car } 2 \quad[\mathrm{~mm}] \\
\end{array}$ & $\begin{array}{r}2 \\
0 \\
-2 \\
\end{array}$ & 㓫 \\
\hline $\begin{array}{l}\text { Date: } 12 \text { September } \\
3197 \text { points total } \\
\text { Car } 1 \quad[\mathrm{~mm}]\end{array}$ & $\begin{array}{r}2 \\
0 \\
-2 \\
\end{array}$ & W \\
\hline $\begin{array}{l}\text { Date: } 26 \text { September } \\
3199 \text { points total } \\
\text { Car } 2 \quad[\mathrm{~mm}]\end{array}$ & $\begin{array}{r}2 \\
0 \\
-2 \\
\end{array}$ & 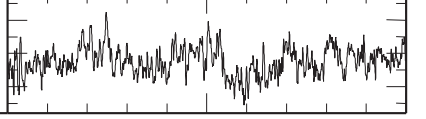 \\
\hline
\end{tabular}

Fig. 12 Example consisting of 11 measured datasets obtained using two inspection cars for the same section of track

constructed using dynamic programming.

When more than two datasets are adjusted, the procedure is basically the same as described above. However, the definition of the evaluation function in (1) changes. As an example, the function is expressed as $F\left(\mathbf{l}_{1: T}, \mathbf{m}_{1: T}, \mathbf{n}_{1: T}\right)$ if there are three datasets. The statistical model simultaneously changes (not discussed here).

\subsection{Browsing through datasets}

Before performing the computation described in Sections 2 and 3, the author examined similarities among the datasets in order to verify the assumption that temporal variations in the gauge are negligible in the adjustment. However, since these 11 datasets had not been adjusted, the differences among them could not be directly computed. The similarities were therefore evaluated using the dynamic time warping distance (DTW distance) [4]. DTW distance is a function of two waveforms, and a shorter DTW distance indicates waveforms with greater similarity. 


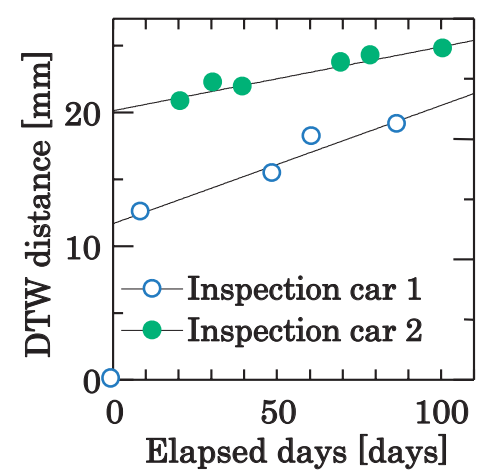

Fig. 13 DTW distance as a function of the number of days elapsed since 17 June

Figure 13 shows a plot of DTW distance between each dataset and the oldest dataset (measured on 17 June) as a function of the number of elapsed days. This figure presents the following two characteristics:

- DTW distances tend to be categorized on the basis of the track inspection car used. This indicates that differences exist among the individual cars.

- DTW distances tend to increase with the number of elapsed days. This indicates the existence of temporal variations in the gauge.

For confirmation of these two characteristics, a dendrogram is also plotted in Fig. 14 from a matrix of DTW distances. Note that the group average method was selected from several available methods for plotting the figure. This dendrogram also exhibits the following two characteristics:

- Differences among individual inspection cars are responsible for the second largest cluster.

- Distances recorded on subsequent inspection days form a small cluster.

These characteristics exhibit the same properties as those indicated in Fig. 13.

In this adjustment, the datasets obtained using Car 2 are selected.

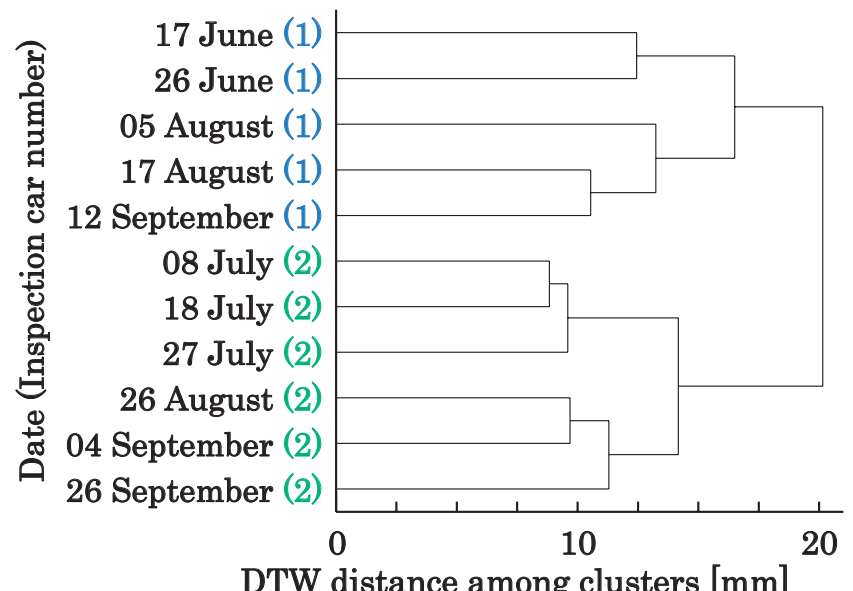

Fig. 14 Dendrogram of DTW distance among datasets shown in Fig. 12

\subsection{Results of adjustment}

Figure 15 displays the shifted sampling locations of the vertical-irregularity datasets, which were inspected

(A) Vertical geometry
before adjustment
26 August $[\mathrm{mm}]$
(B) Vertical geometry
before adjustment
04 September [mm]
(C) Vertical geometry 3
before adjustment
26 September [mm]_3

\begin{tabular}{|c|c|}
\hline $\begin{array}{lr}\text { (B)-(A) } & 0.4 \\
\text { Before adjustment } & 0 \\
\text { (Magnified) [mm } & 0.4\end{array}$ & 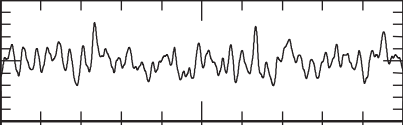 \\
\hline $\begin{array}{l}\text { (C)-(B) } \\
\text { Before adjustment } \\
\text { (Magnified) [mm }]_{-0.4}\end{array}$ & $N^{N a}$ \\
\hline
\end{tabular}

(B)-(A)
$\begin{aligned} & \text { After adjustment } \\ & \text { (Magnified) [mm] }\end{aligned}$
$\begin{aligned} & \text { (C)-(B) } \\ & \text { After adjustment }\end{aligned}$
0

Fig. 15 Vertical geometry datasets (A), (B), and (C) obtained at the same time as the three gauge datasets in Fig. 12. The sampling locations of (A), (B), and (C) are shifted according to the adjustment applied to the gauge datasets.

at the same time as the three datasets selected from the gauge sets in Fig. 12. Although the shifted data-point sequences were constructed independent of the values of the vertical datasets, the amplitude of the difference sequences among the vertical sets seems reasonable.

In addition, the power spectral densities of the difference sequences are also displayed in Fig. 16. The amplitudes of wavelengths around $10 \mathrm{~m}$ decrease after adjustment, indicating that these components seem to arise from the location gaps before adjustment and not from temporal variations in vertical track geometry. A vertical-irregularity dataset originally includes large components around 10-m wavelengths since the measur-

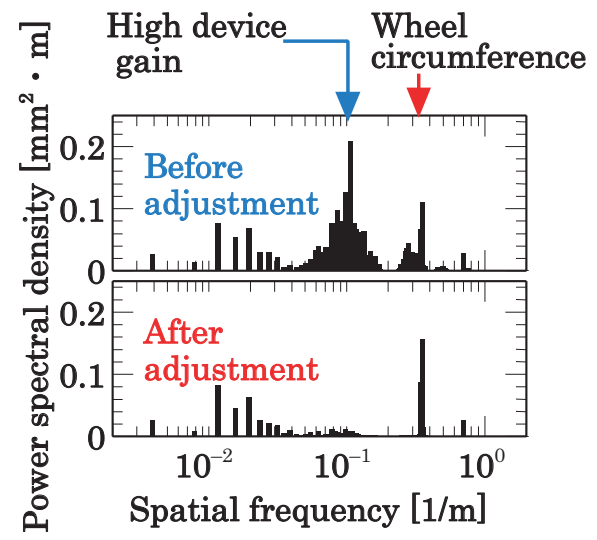

Fig. 16 Power spectral density of the different datasets in Fig. 15 as a function of spatial frequency 
ing device in the track inspection car achieves high gain around $10 \mathrm{~m}$.

Furthermore, in Fig. 16, the spectral component with a wavelength of approximately $2.8 \mathrm{~m}$ is more noticeable after adjustment. This component seems to be derived from the wheel circumference and not from the existing track geometry.

\section{Conclusion}

- Sampling locations for track geometry datasets obtained using a track inspection car can be approximately adjusted by optimization using dynamic programming.

- The unknown parameter introduced by modelling can be identified using the maximum likelihood method.

- Brief analysis performed before adjustment indicates the existence of individual differences among the inspection cars and temporal variations in the gauge. Although these properties have not yet been specified explicitly, they are generally accepted.

- The developed adjustment procedure will be useful in observing variations in track geometry.

\section{Acknowledgements}

The author would like to express her deepest gratitude to Prof. Tomoyuki Higuchi of the Institute of Statistical Mathematics for his valuable guidance and advice.

\section{References}

[1] S. L. Marple, Jr., Digital Spectral Analysis with Applications. Englewood Cliffs, New Jersey: Prentice Hall, pp. 43-44, 1987.

[2] H. Akaike, "Likelihood and the Bayes procedure," in Bayesian Statistics, J. M. Bernardo, M. H. DeGroot, D.V. Lindley and A. F. M. Smith, Eds., Valencia: University Press, pp. 143-166, 1980.

[3] M. Kamiyama, and T. Higuchi, "Adjustment of Nonuniform Sampling Locations in Spatial Data Sets," IEEE Signal Processing Magazine, pp. 47-56, May 2004.

[4] Donald J. Berndt and James Clifford, "Finding Patterns in Time Series: A Dynamic Programming Approach," in Advances in Knowledge Discovery and Data Mining, pp. 229-248, 1996. 